BMJ Quality

\title{
Chasing the Golden Hour - Lessons learned from improving initial neutropenic sepsis management.
}

\author{
Caroline Forde, Paula Scullin
}

To cite: Forde C, Scullin P. Chasing the Golden Hour Lessons learned from improving initial neutropenic sepsis management.. BMJ Quality Improvement Reports 2017;6:u204420.w6531. doi:10.1136/bmjquality. u204420.w6531

Received 11 August 2016 Revised 28 November 2016

\section{CrossMark}

Belfast Health and Social Care Trust, Northern Ireland, UK

Correspondence to Caroline Forde caroline. forde@belfasttrust.hscni.net

\section{ABSTRACT}

Neutropenic sepsis remains a time critical and potentially fatal complication of systemic anti-cancer therapy. A target 'door to needle' time of one hour for first dose empirical intravenous antibiotics continues to be promoted nationally. A baseline audit (June 2011) highlighted shortfalls in care in the Belfast Trust, with only $15 \%$ of patients receiving antibiotics within sixty minutes. A multi-professional group within the Trust was established to try and initiate the improvements in neutropenic sepsis recognition and initial management that were urgently required. A number of strategies have been developed over the last five years. Firstly an integrated care pathway was introduced, which is currently used by nursing and medical staff for patients presenting with suspected neutropenic sepsis, through acute cancer centre assessment areas and emergency departments, as well as inpatients developing neutropenic sepsis. An initial reaudit June 2012 demonstrated improvement (62\% meeting 1hour target), but a subsequent audit, January 2013, was disappointing (only 50\% meeting 1hour target). In response, a new compact, user-friendly care pathway was introduced. A range of other measures have also been subsequently introduced. Patients' care is continually monitored through simple ward based documentation, completed after initial treatment of each neutropenic sepsis episode. A patient group direction facilitates nurse led prescribing and administration of first dose antibiotics. Regular multidisciplinary education sessions and improved access to regional guidelines have also been prioritised. From November 2013, consistently greater than $80 \%$ of patients have met the one hour target. Recent data continues to be encouraging; in July 2016 $100 \%$ of patients received first doses within sixty minutes, in October $95 \%$ of patients. Significant sustained improvements in meeting the sixty minute target have been demonstrated. The combination of measures ensures neutropenic sepsis is considered and basic clinical care delivered quickly and safely, through a co-ordinated standardised approach, to avoid complications.

\section{PROBLEM}

Neutropenic sepsis (NS) remains a frequent complication of cytotoxic chemotherapy causing significant morbidity and mortality. Despite primary and secondary prophylaxis strategies, using colony stimulating factors and antibiotics in high risk patient groups, mortality from untreated NS is still reported to range from $2-21 \% .^{1}$ As the volume of cytotoxic chemotherapy prescribed continues to rise, NS will likely remain a common problem cancer patients receiving systemic anti-cancer therapy (SACT) develop.

Northern Ireland's regional cancer centre for oncology patients is based in the Belfast Trust at the Belfast City Hospital site. A haematology service is also based at the Belfast City Hospital and similar to the oncology service provides chemotherapy for people living in Belfast as well as specialist regional services. Both services have inpatient beds and the majority of patients with suspected NS normally present following contact with the 24 hour chemotherapy helpline to acute oncology/haematology assessment areas. There is no longer an Emergency Department (ED) located at the Belfast City Hospital but patients sometimes also present to EDs at the nearby Royal Victoria Hospital or Mater hospital for initial assessment. Ongoing inpatient care is then normally provided in the Cancer Centre, Belfast City Hospital if feasible.

In response to national recommendations, a Northern Ireland Cancer Network (NICaN) NS guideline was developed and disseminated in 2010. It was aimed at raising awareness of NS and providing simple guidance on initial patient management during the first 48 hours of admission. It included easy to follow clinical algorithms for staff and included the standard of a door to needle time for first dose empirical antibiotics of one hour. There was concern however from staff in the Cancer Centre in Belfast, that there remained a lack of awareness of the guidance and that some patients were receiving suboptimal care with delays in 
recognition and initial treatment noted. No baseline data existed however to assess the quality of NS care being delivered.

This project details the Belfast Trust's multidisciplinary efforts to improve awareness and compliance with the regional NS guidance. In particular the primary project objective was to ensure that consistently greater than $80 \%$ of patients were receiving first dose empirical intravenous antibiotics within sixty minutes of presentation to secondary care with suspected NS. Originally a twelve month timeline was set but as the project progressed the teams focus was to implement sustainable rather than short term interventions.

\section{BACKGROUND}

Management of SACT toxicity became a national clinical priority in 2008 following significant concerns raised by a National Confidential Enquiry into Patient Outcome and Death, reviewing deaths within 30 days of SACT. ${ }^{2}$ Deficiencies in NS care were highlighted, with repeated delays in recognising and initiating appropriate antibiotic treatment noted when reviewing cases. The enquiry recommended emergency admission services be better equipped with the resources to manage NS, including clear clinical pathways for patients, easily accessible local polices and staff training.

In response, the National Chemotherapy Advisory Group in 2009 promoted the development of acute oncology services to respond quickly to patients admitted with SACT complications. ${ }^{3}$ Again clear pathways and protocols for suspected NS were encouraged and a target one hour 'door to needle' time for administration of first dose antibiotics on arrival to hospital was recommended.

The National Institute for Health and Care Excellence (NICE) also urgently developed a clinical guideline for NS management which was published in $2012 .{ }^{4}$ It recognises the wide spectrum of severity of NS, which encompasses a heterogeneous group of patients, ranging from those with uncomplicated fever to septic shock without fever. Use of risk stratification tools, for assisting clinicians in assessing patients' risk of septic complications and choice of ongoing antibiotic treatment is therefore encouraged within 24 hours of presentation to hospital. The focus initially however, remains firmly on prompt assessment and treatment, with the guidance highlighting that suspected NS should be viewed as an acute medical emergency with empiric antibiotic therapy offered immediately.

A target 'door to needle' time is not specifically recommended by NICE, as they noted there were no high quality studies defining an optimal time for antibiotic administration. It is obvious that time to antibiotics and resuscitative measures has greatest impact on patients at greatest risk of septic complications and the implications for low risk patients are less well defined. In the acute care setting however, with front line staff that may not have significant experience managing cancer patients or assessing their risk, many institutions have found it more pragmatic, especially when pursuing local improvement, to set treatment goals that are easily applied to whole patient groups. There therefore has remained considerable support for a one hour 'door to needle' target to aim for, in all patients presenting with suspected NS and this continues to be reflected in many trusts local NS policies.

Widespread agreement persists that NS should continue to be viewed as an important time critical medical emergency, with urgent assessment, resuscitation measures if required and administration of a first dose of broad spectrum empirical intravenous antibiotics, to facilitate rapid identification of high risk patients for whom aggressive measures can be lifesaving.

\section{BASELINE MEASUREMENT}

An initial audit to assess implementation of the regional NS guidance was completed over a six week period in June 2011..$^{5}$ The management of 53 oncology and haematology patients with NS, admitted across the five acute trusts in Northern Ireland was reviewed retrospectively. 53\% (28) of those patients were treated in the Belfast Trust. NS occurred most commonly in breast patients (30\%) and the majority of admissions occurred via emergency departments (ED) and outside of normal working hours.

Unfortunately significant deficiencies in care were noted. Only $15 \%$ (8) of patients presenting with NS received their first antibiotic dose within sixty minutes of presentation to hospital, out of a target of $80 \%$ of cases. Over one third (20) waited over 3 hours from presentation to their first dose. Other shortfalls in management were also highlighted with only $75 \%$ of patients having had blood cultures taken.

The delays in time to first dose antibiotics were in keeping with what was being recognised nationally. A review in 2011 of 27 NHS based audits of NS management $(n=590)$, demonstrated only $26 \%$ of patients received antibiotics within sixty minutes. ${ }^{6}$ Contributing reasons identified for the delays included lack of staff recognition of the time-dependent nature of NS, staffing pressures with varying or unpredictable patient workload in acute areas such as the ED, communication difficulties and lack of timely access to certain antibiotics.

Our baseline results were disappointing and it was agreed there would continue to be a focus on measuring how many patients were receiving their first dose of antibiotics within the one hour target. This was viewed as a clinically important target to aim for but would also serve as a useful general indicator of the overall quality of initial NS care being delivered. It was initially proposed this be performed six monthly, with junior medical staff retrospectively reviewing the clinical notes of oncology and haematology patients who had been admitted with NS, to see if the one hour target had 
been met. The results would be presented and discussed at multidisciplinary audit and quality improvement meetings (See supplementary - Belfast HSC Trust neutropenic sepsis Integrated Care Pathway).

\section{DESIGN}

It was recognised in the Belfast Trust, similar to all acute trusts that both frontline junior medical and nursing staff managing initial NS move between hospitals and specialties, often without extensive knowledge of SACT or its complications. It was therefore agreed these staff groups needed to be better equipped with the resources to consistently recognise and promptly initiate NS treatment.

A multi-professional Belfast Trust group was established initially to develop an Integrated Care Pathway (ICP) for patients presenting with suspected NS. The group involved both junior and senior medical staff, inpatient, unscheduled care and ED nursing staff and a pharmacist, ensuring representation from all speciality areas in which the pathway would be utilised including oncology, haematology, general medicine and the EDs. The group was also supported from leadership within Cancer Services and expert advice provided from the trust's care pathway coordinator. The pathway was to be based on the regional NS guidelines and would aim to ensure a safe, standardised approach to management.

\section{STRATEGY}

Timeline of key project interventions:

2010 - Regional Northern Ireland NS guidelines published

June 2011 - Baseline measurements of Belfast Trust NS practice

July - October 2011 - Multi-professional Belfast Trust NS working group established - Integrated Care Pathway (ICP) trialled and refined in one acute oncology assessment area

November 2011 - Original ICP introduced across the Trust

January 2014 - Shortened ICP introduced across the Trust

September 2014 - Continuous data collection commenced across the Trust

September 2015 - Patient Group Direction for IV Tazocin first introduced in haematology followed by oncology

December 2015 - Acute oncology guidelines launched including NS guidelines (and available as a mobile phone app)

Project interventions

Introduction of original Integrated Care Pathway (ICP)

The ICP document was designed with the aim of clearly highlighting NS as a time critical condition, emphasising for nursing and medical staff the essential management steps required within the patient's first hour of care. The pathway was to be commenced for any oncology or haematology patient receiving chemotherapy within the last six weeks who presented unwell or febrile. It was to be used in EDs and acute oncology/ haematology assessment areas, as well as for inpatients who developed NS.

For the first PDSA cycle, the ICP was initially tested in one acute assessment area for oncology patients, where it was refined with small tests of change over several months following feedback from medical and nursing staff.

For the second PDSA cycle, the ICP was formally launched for use across oncology, haematology and EDs in November 2011 with training sessions provided for all staff members likely to use the documentation. An initial review of 17 patients' management was undertaken approximately 7 months after pathway implementation in June 2012. Encouragingly it demonstrated improvements, with $62 \%$ of patients receiving antibiotics within sixty minutes and $62 \%$ of patients having an ICP completed. It was therefore agreed that the ICP should continue to be used and promoted.

Introduction of the shortened ICP

A subsequent follow up review of 25 patients over a four week period in January 2013 was disappointing however, with the number of patients meeting the one hour door to needle target falling to only $50 \%$.

In response, for the third PDSA cycle, further user feedback was sought from both medical and nursing staff in all areas where the ICP was being used and a more compact, user friendly pathway started to be developed. To encourage compliance, both the triage nursing assessment and medical assessment were simplified to ensure the documentation was as easy and quick to complete as possible. A pragmatic 'ABCD' checklist was incorporated to ensure appropriate observations, monitoring, essential investigations and first dose antibiotics were commenced.

As the new shortened ICP was being trialled on a small scale, a follow up review of 33 patients over a four week period in November 2013 was completed which demonstrated improved practice with $80 \%$ of patients meeting the 1 hour door to needle target. Only $62 \%$ of patients had an ICP completed but interestingly in the majority of cases $(83 \%)$ not meeting the 1 hour target an ICP had not been utilised.

For PDSA cycle 4, the new version of the shortened ICP was launched across the Trust in January 2014, with further staff training sessions. Feedback was very positive with many commenting the shorter documentation was much preferred. A follow up audit in July 2014 showed continued improvement. The shorter ICP was being utilised more than it previously had been ( $89 \%$ uptake) and $86 \%$ of patients were now meeting the 1 hour door to needle target.

Introduction of continuous data collection The aim initially had been to audit practice approximately six monthly but there was an awareness that these 
audits were difficult to deliver in a timely manner. They were time consuming for a new junior doctor to complete on each occasion and there was often a several month delay between the month's admissions that were being reviewed and the results being fed back to staff. It was also proving challenging to maintain staff interest and momentum in between audit results being discussed. In response, for PDSA cycle 5, a ward based document was developed initially in haematology and then introduced to oncology in September 2014 to try and ensure continued prioritisation of timely NS care. Ward nursing staff now immediately review all aspects of a patient's care by completing this simple ward documentation. (Supplementary File 2) Monthly outcomes are reported at SACT and governance meetings and fed back to staff. Cases failing the sixty minute target are then reviewed to highlight areas for improvement. Results are displayed on ward information boards to inform staff, patients and the public of the latest results. Generally since the introduction of continuous data collection the number of patients meeting the one hour target has been greater than prior to it being introduced.

Patient Group Direction (PGD) for nurse led prescribing and administration of first dose intravenous antibiotics

Speciality nursing staff are now also able to administer first dose intravenous Tazocin, to patients with suspected NS prior to medical review, with the aim of further reducing time to antibiotics. This was the focus of the final PDSA cycle (cycle 6), with the PGD first introduced in haematology from September 2015 and more recently in oncology in 2016. High levels of patients meeting the one hour target have been maintained since this option was introduced, which has proven a popular strategy with staff.

Additional multidisciplinary strategies have also been implemented to raise awareness of the importance of early recognition of NS and improve initial management.

\section{Multidisciplinary Education}

Training centred on both NS and use of the ICP has been incorporated into routine induction for both haematology and oncology medical and nursing staff. 24 hour chemotherapy helpline training reminds staff of the importance of ensuring patients present their 'alert cards' if being advised to attend an ED and ringing the ED in advance to explain the patient has suspected NS. Updates and latest results are also provided to staff at multidisciplinary grand round meetings, audit and quality improvement meetings. At several trust public engagement events poster presentations have also highlighted to staff, patients and the public the trust's prioritisation of high quality NS care and progress made to date.

Acute Oncology guidelines

As part of acute oncology service development NICaN published Acute Oncology guidelines in December
2015, containing the regional NS guidelines. These guidelines are available as a free mobile phone app making them easily accessible to a wide range of staff working across different specialities. Junior medical staff have been particularly enthusiastic about this resource, enjoying the ease at which they can quickly check regional guidance and reporting that it better equips them to deliver evidence based care consistently.

\section{RESULTS}

A summary of results over the last five years, from June 2011 to October 2016 is presented in Figure 1.

Post implementation of the abbreviated ICP, compliance with the documentation started to improve and improvements were also noted in the number of patients meeting the one hour target. Introduction subsequently of the ward based continuous data collection and patient group direction for antibiotic administration has helped maintain high rates of patients receiving antibiotics promptly. For nearly three years now, from November 2013 , we have consistently had greater than $80 \%$ of patients meeting the one hour target. Recent results continue to be encouraging; in July $2016100 \%(n=14)$ of patients received their first dose within sixty minutes, in October $95 \%$ of patients $(n=19)$. Median time to first dose antibiotics was 40 minutes in October 2016.

Additional aspects of initial care have also been improved. Routine observations were performed in $100 \%$ of patients in October $2016 \quad(\mathrm{n}=19)$ compared with $72 \%$ in June 2011. Routine bloods including full blood count, biochemistry and CRP were completed in $100 \%$ of patients in October compared with $92 \%$ in the 2011 audit. Encouragingly 100\% of patients also had blood cultures compared to $75 \%$ in 2011. In October $100 \%$ of patients had an appropriate examination documented and appropriate further investigations undertaken if clinically indicated compared to $75 \%$ and $79 \%$ respectively in 2011.

Antibiotic prescriptions are also carefully monitored. To reflect NICE guidance our regional guidelines were amended in January 2014, so that Tazocin monotherapy is the first line choice in non-penicillin allergic patients, with Gentamicin reserved for signs of severe sepsis. From February to October 2016 for example, all first line antibiotics prescribed in oncology and haematology patients with NS have been deemed to be appropriate.

Our data provides evidence that the introduction of a range of simple, multidisciplinary measures including an integrated care pathway, continuous data collection and patient group direction for first dose antibiotic administration can result in sustained improvements in compliance with regional NS guidelines and timeliness of first dose antibiotics.

\section{LESSONS AND LIMITATIONS}

Attitudes, approaches and delivery of initial NS care continues to vary nationally. Several institutions, similar to 


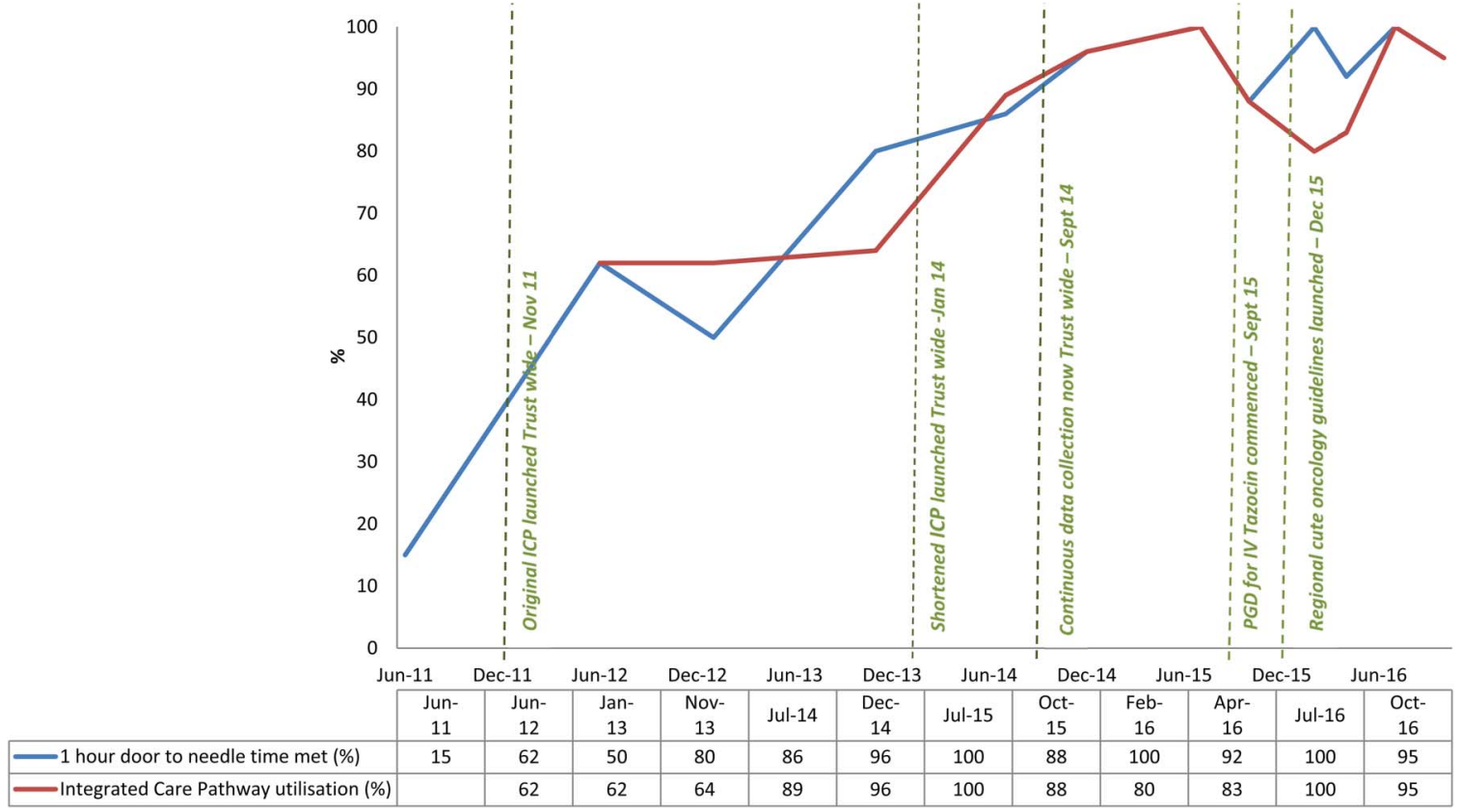

Figure 1 Summary results of initial neutropenic sepsis management in Belfast Trust Oncology and Haematology patients from June 2011 to present.

us have shared publically both successes and challenges in delivering care. Most of these accounts have however been in poster presentations at national conferences, with only abstracts available, making it difficult therefore to ascertain the exact nature of the interventions trialled, their outcomes, sustainability and how they may be replicated. It is evident nonetheless, that a wide range of interventions nationally have been successfully implemented in cancer centres, cancer units and general district hospitals.

Williams (2014) described a number of interventions introduced in Cheltenham General Hospital Oncology centre, including a multidisciplinary clinical pathway and education programme based on the sepsis six care bundle and a patient group direction for nurse led initiation of antibiotics. ${ }^{7}$ They reported only $32 \%$ of 26 patients over a 2 week baseline audit received antibiotics within one hour, which improved to $97 \%$ over a 2 week reaudit following implementation of their changes.

Acute oncology (AO) services too have played an important role in District General Hospitals for promotion of NS care to generally non-specialist clinical teams. Sephton (2014) highlighted positive developments in NS management in Yeovil, a district general hospital in Somerset. $^{8}$ A range of measures were introduced through an AO team, with similar strategies employed to ourselves, including weekly electronic auditing of performance with feedback to staff and implementation of an education programme and patient group direction for antibiotic administration. They reported an increase in patients meeting the one hour target from $67 \%$ in October 2013 to 94\% in March 2014.

Ghosh (2015) also reflected on several years' experience of door to needle times in a district general hospital in Great Yarmouth, James Paget University Hospital. $^{9}$ They reported the introduction of an AO service had improved door to needle times and also had interestingly introduced an annual NS awareness day and a stamp to record critical times in the patient's care pathway for medical notes.

Other services too have been refreshingly open about the ongoing challenges they encounter trying to deliver initial NS care. Palethorpe (2013) noted in the Birmingham hospital trust that despite the introduction of an $\mathrm{AO}$ service, still only a quarter of patients received antibiotics within one hour. ${ }^{10}$

In the Belfast Trust, a simple, concise ICP ensures NS is considered and essential clinical care can be delivered systematically and quickly through a co-ordinated approach, allowing identification of high risk patients and avoiding septic complications. Ward based documentation for continuous data collection has provided a simple, innovative way to continually highlight the importance of NS, review progress and offer encouragement. A patient group direction facilitating nursing staff to commence patients at triage on the NS care pathway and administer first dose antibiotics has further reduced delays and empowered front line nursing staff to be more independent in initiating and delivering patient care.

There were several important lessons learnt during the course of this project which have already influenced other quality improvement work subsequently undertaken locally. This project was commenced five years ago and the team's understanding of quality improvement methodology has significantly developed since then. In retrospect, at the beginning of this project in particular, multiple, smaller scale, shorter PDSA cycles would have 
been beneficial and facilitated more rapid progress and improvements in care. More frequent data collection from the outset, similar to what we are currently undertaking, would have facilitated more rigorous evaluation of the changes being tested, guided decision making and likely produced a more suitable original care pathway. It is acknowledged, that initially data collection was too infrequent, often several months after an intervention had been implemented and on the basis sometime of only one set of results, which may or may not have been representative of ongoing practice, changes were sought. The introduction of continuous data capture, which staff have not found too burdensome, was important not only for providing a gentle ongoing reminder of the importance of NS but also facilitating a continuous up to date understanding of current practice.

It was nevertheless useful to have all key stakeholders involved from the outset of this project to gain 'buy in' and cooperation. There was clear leadership and support for change from senior trust management and this area of work has continued to be prioritised over time. Sustainability, like for many quality improvement initiatives, has continued to be the biggest challenge. It was strongly felt early on that a multifaceted approach would be beneficial and the shortening of the ICP and introduction of continuous data capture have both been felt to be crucial components to this project's success. A committed and sustained effort, involving the whole multidisciplinary team has been fundamental for this projects longevity, with 'champions' within each speciality particularly helpful, to not only lend the project credibility but to also continue to drive progress forward. A robust regular multidisciplinary education programme has also been essential to prevent the project from 'fizzling out', as has an active willingness to seek out, listen and act on feedback to make interventions more user friendly. Encouragement and immediate feedback of positive results to front line staff, celebrating success, has enabled staff engagement and ownership of both the ICP and ward documentation for continuous data collection.

The location of where patients present continues to influence ICP utilisation and time to antibiotics. Shortest time to antibiotics occurs when inpatients develop NS or when experienced staff in the acute oncology/haematology assessment areas assess patients. The presence of specialist staff and workloads and presence of indwelling lines all also continue to influence time to antibiotics. The ICP documentation is not as well utilised in patients presenting through EDs, principally as they have their own sepsis tool based on the sepsis six and there has understandably therefore been reluctance for additional documentation. Over time however, the number of NS patients presenting through an ED has dramatically reduced with expansion of oncology/haematology unscheduled care provision. The greater challenge of meeting the one hour target in EDs is well recognised and has been reflected in other institutions reports. For example Milverton (2013) discussing the experiences at Russells Hall General Hospital found significantly longer delays to first dose antibiotics when patients presented through the ED (26\% within the hour) and the Emergency and accident unit $(0 \%$ within the hour) compared to when patients where admitted through haematology/oncology triage $(81 \%$ within the hour). ${ }^{11}$ Longer delays in patients presenting through an ED most commonly arise however in our experience in patients later deemed low risk; often having a single fever at home but on triage no signs or symptoms suggestive of sepsis and therefore it is unlikely a longer delay to first dose antibiotic in these patients influences outcome. Ongoing discussion with the ED remains a priority however to continue to optimise care, particularly for high-risk patients who may present through this route.

It has also not been possible to determine if the changes we have implemented have had any significant impact on mortality from NS due to the small number of deaths yearly from NS. Any oncology deaths from NS are discussed at the monthly regional morbidity and mortality meeting where care is carefully peer reviewed and suggestions for improvements discussed and disseminated widely. Future work in the Belfast Trust will now focus on strategies to encourage earlier patient presentation and optimising management of high risk NS patients.

\section{CONCLUSION}

For commonly encountered clinical problems the sharing of simple pragmatic interventions that allow guidelines to be embedded into routine practice should be encouraged. The interventions utilised in this quality improvement programme could be easily adapted to suit other trusts NS guidelines or other protocols requiring safe, timely and efficient care to be delivered.

Consistently over $80 \%$ of patients in the Belfast Trust for approximately three years have now received first dose antibiotics for NS within sixty minutes of presentation compared to a baseline of $15 \%$ in 2011. These results compare favourably with published national data and significant improvements have also been seen in all aspects of initial care, with increased compliance with local NS guidelines. The range of measures introduced ensures a safe, standardised approach to initial management, improving the quality of NS care patients' receive.

Acknowledgements With thanks to Debbie McKelvey (Acting Oncology Inpatients Assistant Service Manager) Belfast Health and Social Care Trust who significantly contributed to the development of this quality improvement project.

Thanks also to those currently co-ordinating ongoing monitoring of this project - Joanne Stewart and Angela Walsh (Haematology) and Nicola Shannon, Monica Donovan, Helen Poots, Janine Palmer and Viveen Lees (Oncology).

The adoption, feedback and support from all medical and nursing staff in the Belfast Trust on the range of measures introduced as part of this 
neutropenic sepsis quality improvement project has been invaluable and much appreciated.

Declaration of interests Nothing to declare.

Ethical approval Ethical approval for this project was not required due to it being a quality improvement project aimed at improving delivery of initial neutropenic sepsis care in the Belfast Health and Social Care Trust, in line with local trust policy.

Open Access This is an open-access article distributed under the terms of the Creative Commons Attribution Non-commercial License, which permits use, distribution, and reproduction in any medium, provided the original work is properly cited, the use is non commercial and is otherwise in compliance with the license. See:

- http://creativecommons.org/licenses/by-nc/2.0/

- http://creativecommons.org/licenses/by-nc/2.0/legalcode

\section{REFERENCES}

1. Herbst C, Naumann F, Kruse EB et al. Prophylactic antibiotics or G-CSF for the prevention of infections and improvement of survival in cancer patients undergoing chemotherapy (Review) 2009. Cochrane Database Syst Rev 21:CD007107.

2. National Confidential Enquiry into Patient Outcome and Death. For better, for worse: A review of the care of patients who died within 30 days of receiving systemic anti-cancer therapy. 2008 http://www.ncepod.org.uk/2008report3/Downloads/SACT_ summary.pdf

3. National Chemotherapy Advisory Group. Chemotherapy Services in England: Ensuring quality and safety. 2009 http://webarchive. nationalarchives.gov.uk/20130107105354/http:/www.dh.gov.uk/prod_ consum_dh/groups/dh_digitalassets/documents/digitalasset/dh_ 104501.pdf

4. National Institute for Health and Care Excellence. Neutropenic sepsis: prevention and management of neutropenic sepsis in cancer patients. 2012 https://www.nice.org.uk/Guidance/CG151

5. Scullin P, Hurwitz J. GAIN (Guidelines and Implementation Network). A Regional Multi professional Audit of the Management of Neutropenic Sepsis in Adults Receiving Systemic Anti-Cancer Therapy (SACT). $2012 \mathrm{https://rqia.org.uk/RQIA/files/8e/}$ 8e2b97e3-58dc-4d77-b1cd-08312cec5742.pdf

6. Clarke RT, Warnick J, Stretton K, Littlewood TJ. Improving the immediate management of neutropenic sepsis in the UK: lessons from a national audit. Br J Haematol (2011); 153:773-9.

7. Williams A, Candish C, Ayrton C et al. Reducing the Door to Needle Time for Antibiotics in Suspected Neutropenic Sepsis Using a Dedicated Clinical Pathway. Presented at the Royal College of Radiologists Clinical Oncology Audit meeting, London, UK, 2014.

8. Sephton M, Lutyens R, Young S et al. Improving door-to-needle time for suspected neutropenic sepsis in a District General Hospital. Presented at the National Cancer Research Institute cancer conference, Liverpool, UK, 2014.

9. Ghosh N, Han M, Dernedde U. Neutropenic Sepsis Door-to-needle time audit over the last 6 years. Presented at the National Cancer Research Institute cancer conference, Liverpool, UK, 2015.

10. Palethorpe M, Thompson J, Kilhara S. Management of febrile neutropenia in an Acute Oncology Service: are we meeting the challenge? Presented at the National Cancer Research Institute cancer conference, Liverpool, UK, 2013.

11. Milverton L, Hoyte H. Neutropenic sepsis-Door to needle time. Presented at the National Cancer Research Institute cancer conference, Liverpool, UK, 2013. 